\title{
Long non-coding RNA PTCSC3 inhibits human oral cancer cell proliferation by inducing apoptosis and autophagy
}

\author{
Hongmei Zhang ${ }^{1}$, Jiang Wang ${ }^{2}$, Wenxing Xun ${ }^{1}$, Jia Wang ${ }^{3}$, Wei Song ${ }^{1}$, Xiaoxia Wang ${ }^{4}$
}

\author{
${ }^{1}$ Department of Stomatology, the Second Affiliated Hospital of Air Force Medical \\ University, Xi'an, China \\ ${ }^{2}$ Department of Neurosurgery, the First Affiliated Hospital of Air Force Medical \\ University, Xi'an, China \\ ${ }^{3}$ Department of Ultrasound Diagnostics, the Second Affiliated Hospital of Air Force \\ Medical University, Xi'an, China \\ ${ }^{4}$ Department of Dermatology, the Second Affiliated Hospital of Air Force Medical \\ University, Xi'an, China
}

Submitted: 21 February 2020; Accepted: 17 March 2020;

Online publication: 16 June 2020

Arch Med Sci 2021; 17 (2): 492-499

DOI: https://doi.org/10.5114/aoms.2020.95572

Copyright @ 2020 Termedia \& Banach

\section{Abstract}

Introduction: Long non-coding RNAs (IncRNAs) have gained increased attention due to the discovery of their roles in cancer-related processes. LncRNA PTCSC3 has been shown to have tumour-suppressive effects in thyroid cancer and glioblastoma. This study investigated the role of IncRNA PTSC3 in human oral cancer.

Material and methods: Cell viability was determined by MTT assay. The induction of apoptosis was confirmed by 4',6-diamidino-2-phenylindole (DAPI) and Annexin V/PI assays. Ultrastructural analysis was performed by electron microscopy. Transwell assay was used to monitor the invasion of oral cancer cells. Results: The results revealed significant $(p<0.05)$ suppression of PTCSC3 expression in human oral cancer tissues and cell lines. The overexpression of PTCSC 3 caused a significant $(p<0.05)$ decline in the proliferation of the human oral cancer cells via induction of apoptotic cell death which was accompanied by remarkable enhancement of Bax and suppression of $\mathrm{Bcl}-2$. The electron microscopic analysis showed the development of autophagic vesicles in both the SCC-1 and SCC-9 cells indicative of autophagy. The western blotting analysis showed that PTCSC 3 overexpression caused a remarkable increase in LC3B-I and Beclin 1 expression. PTCSC 3 overexpression caused a significant $(p<0.05)$ decrease in invasion of the human SCC-1 and SCC- 9 oral cancer cells. The invasion of the SCC- 1 and SCC-9 cells was inhibited by $62 \%$ and $69 \%$ respectively.

Conclusions: Overall, the evidence suggests that IncRNA PTCSC 3 acts as a tumour suppressor in human oral cancer and suppresses oral cancer proliferation via induction of apoptosis and autophagy.

Key words: IncRNA, apoptosis, PTCSC3, oral cancer, autophagy.

\section{Introduction}

The human genome and transcriptome studies have revealed that around $85 \%$ of the human genome is transcribed. Nonetheless, it is intriguing that a very low percentage of the transcribed genes code for proteins, indicating that the majority of RNA transcripts are not trans-

\author{
Corresponding author: \\ Hongmei Zhang \\ Department \\ of Stomatology \\ the Second Affiliated \\ Hospital of Air Force \\ Medical University \\ 1 Xinsi Road \\ 710038 Xi'an, China \\ Phone/fax: \\ +86-029-84777375 \\ E-mail: zzhmei0911@163.com
}


lated and are therefore non-coding [1]. These non-coding RNA molecules are categorised into microRNAs (around 21 to 24 nucleotides) and long non-coding RNAs (more than 200 nucleotides) [2]. The microRNAs have received much attention and have been extensively studies and many of their cellular and physiological functions have been revealed $[3,4]$. Over the last decade or so, studies performed on IncRNAs have revealed their functional association with human diseases such as cancer; for example, aberrant expression of IncRNAs has been shown to be linked to the development of colorectal carcinoma [5], hepatocellular carcinoma [6], breast cancer [7] and glioblastoma [8] to name a few. The IncRNAs have shown involvement in cancer-related processes such as growth, cell death, angiogenesis and metastasis [9]. Papillary thyroid carcinoma susceptibility candidate 3 (PTCSC3) is an important IncRNA and was initially shown to suppress the proliferation and metastasis of thyroid cancer via modulation of the S100A4 pathway [10]. Recently, the role of PTCSC 3 was also revealed in glioblastoma. It was found that PTCSC 3 inhibits the wnt/ $\beta$-catenin signalling cascade to suppress the growth and invasion of glioblastoma [11]. Nonetheless, the role of PTCSC3 is still largely unknown in other human cancers. This study was therefore designed to investigate the role of PTCSC3 in human oral cancer and attempts were made to explore the underlying molecular mechanisms. Being one of the serious health issues, oral cancer together with pharyngeal cancer is ranked as the $6^{\text {th }}$ most prevalent cancer type across the globe [12]. Annually 0.27 million oral cancer cases are detected throughout the world and most of these cases are reported from the developing countries [13]. The incidence of oral cancer varies geographically with very high frequency in South and Southeast Asia. Generally oral cancer has been shown to be more prevalent in men than in women [14]. The oral cancer risk increases with age and most oral cancers are detected in people above the age of 50 years. However, oral cancer may also be found in younger people. It has been reported that $6 \%$ of the oral cancer cases are reported in people below the age of 45 years [15]. The 5-year survival rate for oral cancer is around $5 \%$, which is considered very poor. The patients who survive after successful treatment of oral cancer face some severe consequences of the treatment such as the appearance and function. They have severe issues in eating and speaking, which result in depression and malnutrition [16]. Therefore, there is tremendous urgency to development efficient chemotherapy and potent molecular therapeutic targets for the successful management of oral cancers. In the present study we show that IncRNA PTCSC3 is significantly downregulated in human oral cancer cells and its overexpression causes inhibition of oral cancer proliferation via induction of apoptosis and autophagy.

\section{Material and methods}

\section{Tissues, cell lines and transfection} of cancer cells

The oral cancer tissues and the normal adjacent tissues were obtained at the Second Affiliated Hospital of Air Force Medical University, Xi'an, China after informed consent from the patients. The cell lines (SCC-4, SCC-9, SCC-15 and SCC-25) along with a normal (EBTr) cell line were procured from ATCC, USA. The culturing of cell lines was performed using Dulbecco's modified Eagle's medium (DMEM, Thermo Scientific). The cell lines were maintained in a $\mathrm{CO}_{2}$ incubator at $37^{\circ} \mathrm{C}$ with $5 \% \mathrm{CO}_{2}$ concentration and relative humidity of $98 \%$.

\section{Reverse transcription-quantitative polymerase chain reaction ( $R T-q P C R)$}

The total RNA was extracted from the oral cancer cell lines and tissue line with the assistance of RNeasy kits (Qiagen, Inc., Valencia, CA, USA). To reverse transcribe the cDNA, Omniscript RT (Qiagen, Inc.) was employed using $1 \mu \mathrm{g}$ of the extracted RNA. The cDNA was then used as a template for RT-qPCR analysis with the assistance of the Taq PCR Master Mix kit (Qiagen, Inc.) according to the manufacturer's protocol. The cycling conditions were as follows: $95^{\circ} \mathrm{C}$ for $20 \mathrm{~s}$, followed by 40 cycles of $95^{\circ} \mathrm{C}$ for $15 \mathrm{~s}$, and $58^{\circ} \mathrm{C}$ for $1 \mathrm{~min}$. GAPDH was used as an internal control and the relative quantification $\left(2^{-\Delta \Delta C a}\right)$ method was used to evaluate the quantitative variation between the samples.

\section{Transfection}

To stably transfect the oral cancer cells with NC and pcDNA-PTCSC3 (GenePharma, Shanghai, China; 10 pmol), Lipofectamine 2000 (Thermo Scientific) was used and the user guidelines were followed.

\section{Cell viability assay}

Cell viability of the transfected oral cancer cells was determined by MTT assay. Briefly, transfected SCC- 1 and SCC-9 cells were placed in 96-well microplates bearing $100 \mu \mathrm{l}$ of RPMI 1640 medium with 400,000 cells each well and FBS (5\%). Antibiotics were also supplemented to each well plate followed by pre-culturing of both cell lines overnight at $37^{\circ} \mathrm{C}$ in a $\mathrm{CO}_{2}(5 \%)$ humidified incubator. Afterwards, MTT stock solution of $50 \mu \mathrm{l}$ with con- 
centration of $2.5 \mathrm{mg} / \mathrm{ml}$ in culture medium was added to each well and subjected to incubation for an additional $1 \mathrm{~h}$. DMSO was used to dissolve the formazan crystal formed from MTT. Finally, the absorbance was determined with an automatic microplate reader (LKB 5060-006 Microplate Reader, Austria) at $540 \mathrm{~nm}$ wavelength.

\section{Transmission electron microscopic (TEM) analysis}

Autophagy in transfected SCC-1 and SCC-9 cancer cells was investigated via transmission electron microscopy (TEM) analysis. Cells were harvested at $80 \%$ growth confluence, and then subjected to trypsinization. Afterwards, cells were first fixed by glutaraldehyde $(2 \%)$ in phosphate buffer $(0.1 \mathrm{M})$ followed by post fixation with osmium tetroxide (1\%). Cells were then exposed to ethanol embedded in resin followed by cutting of thin sections with an ultramicrotome. Finally, these sections were examined on the transmission electron microscope (Zeiss CEM 902) linked to a digital camera for capturing different fields.

\section{Analysis of nuclear morphology}

The 4',6-diamidino-2-phenylindole (DAPI) staining was performed to examine the nuclear morphology of the transfected SCC-1 and SCC-9 cells. Briefly, the cells were cultured in 6-well plates for $24 \mathrm{~h}$ at $37^{\circ} \mathrm{C}$. About $20 \mu \mathrm{l}$ of cultured cells were loaded on glass slides for DAPI staining. Finally, slides were covered with coverslips and examined under a fluorescent microscope.

\section{Annexin V/PI assay}

To determine the percentage of apoptotic cells, the cultured cells were stained with Annexin VFITC and examined by flow cytometry. For that the FITC Annexin V Apoptosis Detection Kit I (BD Biosciences) was used in accordance with provided guidelines. In brief, sub-culturing of transfected SCC- 1 and SCC- 9 cells at $2.5 \times 10^{4}$ concentration was performed in 6-well plates. Thereafter, both treated cell types were harvested and subjected to PBS washing twice followed by resuspension in $1 \times$ binding buffer. Aliquots of $10^{4}$ cells were subjected to FITC Annexin $\mathrm{V}$ and PI (5 $\mu \mathrm{l}$ each) staining. Finally, a flow cytometer (BD Biosciences, United States) was used to examine each sample.

\section{Transwell cell invasion assay}

The transwell chambers containing polycarbonate members with a pore size of $8 \mu \mathrm{m}$ were put into the six-well plates. The I-type collagen $(10 \mu \mathrm{g} / \mathrm{ml})$ was used for the coating of the lower transwell compartment and subsequently dried. About $200 \mathrm{ml}$ of transfected cells were inoculated in the Matrigel coated upper compartment at the density of $1.5 \times 10^{5}$ cells $/ \mathrm{ml}$. The lower compartment was filled with $800 \mu$ of medium containing FBS (20\%). The whole system was subjected to incubation at $37^{\circ} \mathrm{C}$ for $24 \mathrm{~h}$. The cells that invaded through the membrane with Matrigel were stained with crystal violet and photographed under an inverted fluorescence microscope.

\section{Western blotting assay}

To examine the protein expressions in transfected SCC-1 and SCC-9 cells, western blotting assay was performed. Briefly, the cells were lysed and lysates were analysed through BCA assay for protein quantification. Around $40 \mu \mathrm{g}$ of proteins from each sample were separated by SDS-PAGE and electrophoretically transferred to nitrocellulose membranes (Bio-Rad Laboratories, Hercules, United States). These membranes were then blotted with primary antibodies of Bax, Bcl-2, LC3-I, LC3-II and Beclin-1 (Santa Cruz, CA, USA) having 1 : 1000 dilution. Thereafter, secondary antibody treatment was performed at $4^{\circ} \mathrm{C}$ overnight. Finally, an enhanced chemiluminescence reagent (Amersham, Piscataway, NJ, United States) was used for determination of protein bands.

\section{Statistical analysis}

The experiments were performed in triplicate and expressed as mean \pm SD. Student's $t$ test and one-way ANOVA followed by Tukey's test were used for statistical analysis. $P<0.05$ was considered to indicate a statistically significant difference.

\section{Results}

\section{Oral cancer is associated with suppression of IncRNA PTCSC3}

To gain insights about the transcript profile of PTCSC3 in human oral cancer, qRT-PCR was performed. The results showed that PTCSC3 is significantly $(p<0.05)$ suppressed in oral cancer tissues relative to normal adjacent tissues (Figures $1 \mathrm{~A}, \mathrm{~B}$ ). Next, the assessment of the expression profile of PTCSC3 was also done in normal and oral cancer cell lines. The expression of PTCSC 3 was found to be significantly $(p<0.05)$ suppressed in all the oral cancer cell lines relative to the normal cell line. The downregulation was found to be around 5.9-fold. The highest downregulation was found to be lowest in SCC-1 and SCC-9 cell lines. These two cell lines were selected for further experimentation. 
A

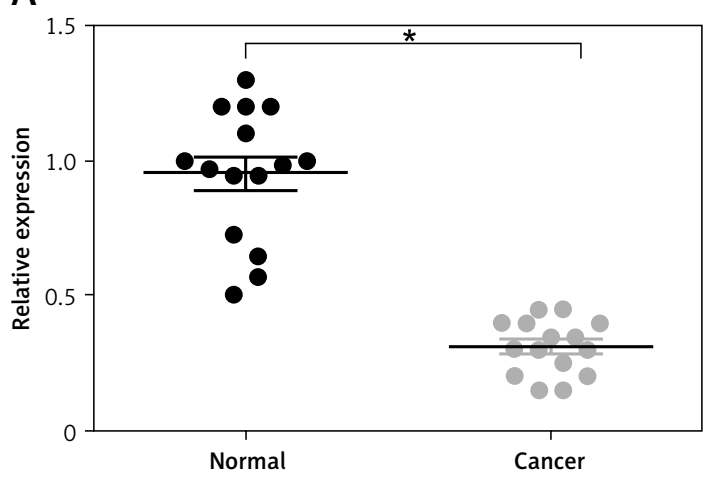

C

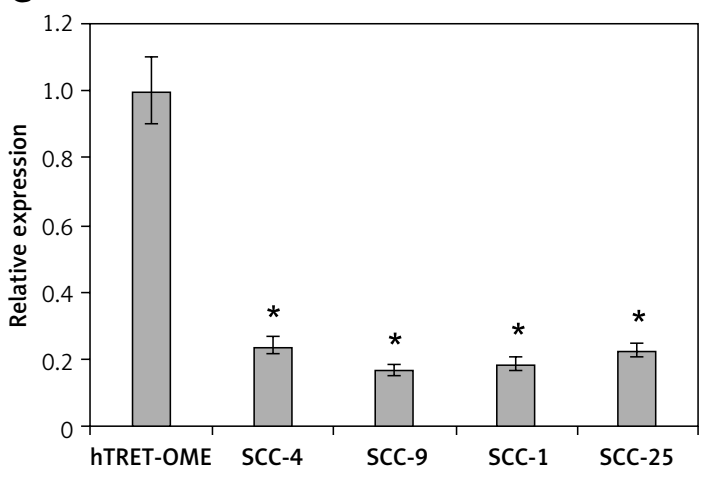

B

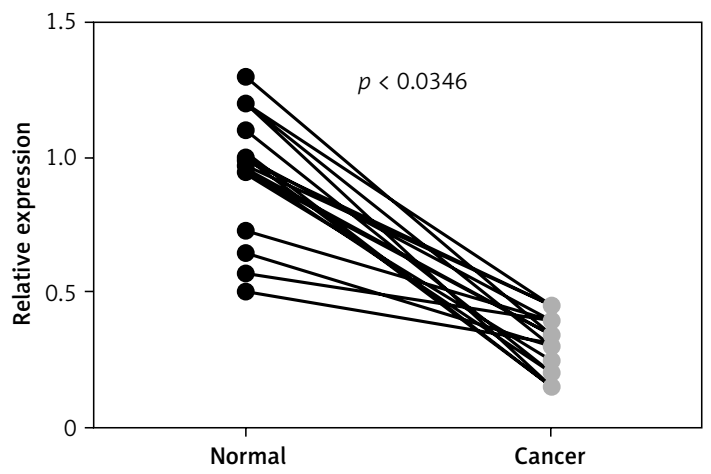

Figure 1. LncRNA-PTCSC3 is downregulated in human oral cancer cells. A - Relative expression of PTCSC3 in oral cancer and normal adjacent tissues. B - Pair-wise expression analysis of oral cancer and normal adjacent tissues. $\mathbf{C}$ - Relative expression of PTCSC3 in normal and oral cancer cell lines. Individual experiments were performed in triplicate and the results shown as mean $\pm \mathrm{SD}\left({ }^{*} p<0.05\right)$
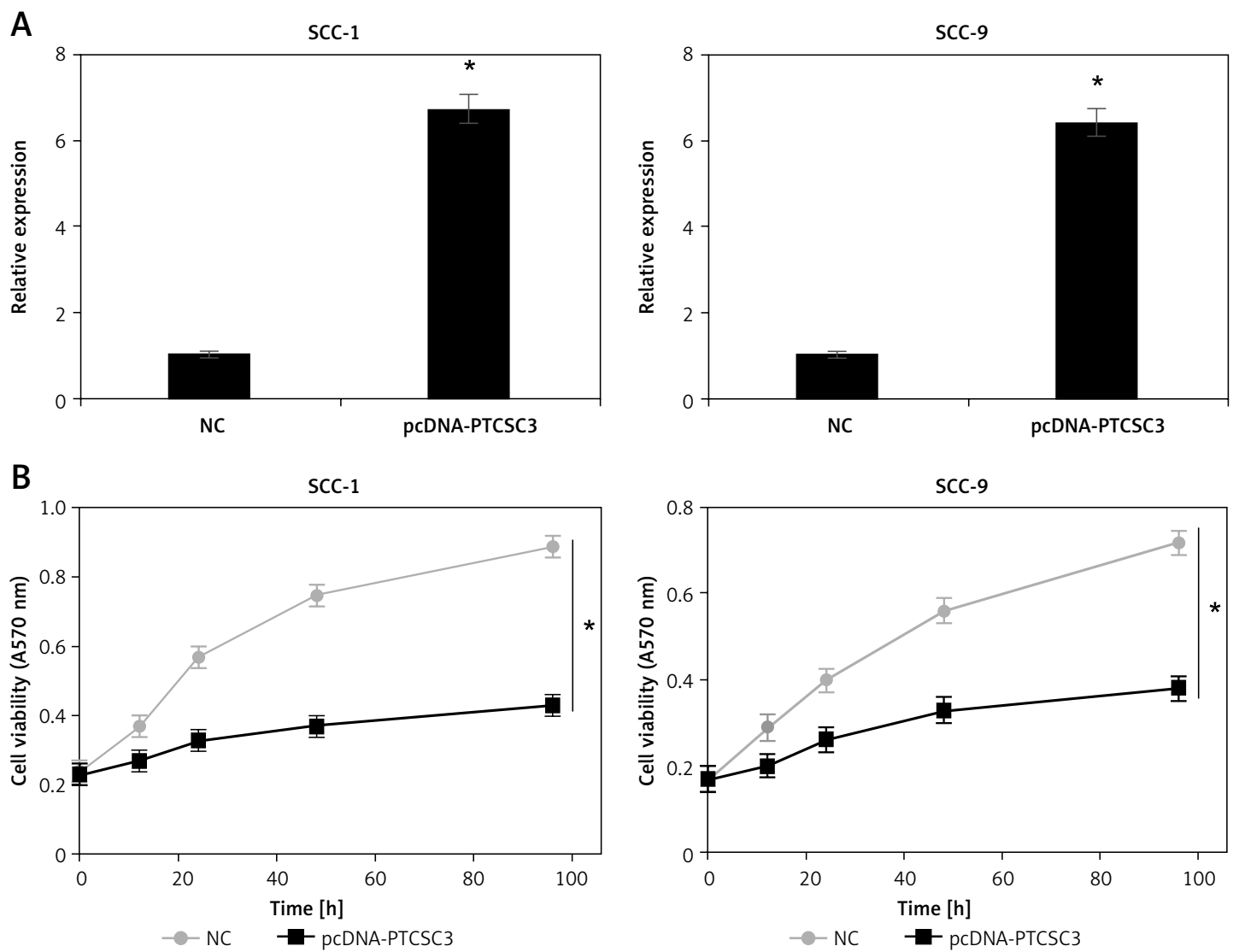

Figure 2. PTCSC3 inhibits viability of the oral cancer cells. A - Expression of PTCSC3 in NC and pCDNA-PTCSC3 transfected SCC-1 and SCC-9 oral cancer cells. B - Cell viability of NC and pCDNA-PTCSC3 transfected SCC- 1 and SCC-9 oral cancer cells. The experiments were performed in triplicate and the results expressed as mean \pm SD $\left.{ }^{*} p<0.05\right)$ 


\section{LncRNA PTCSC3 induces apoptosis in oral} cancer cells

Next, PTCSC3 was overexpressed in SCC -1 and SCC-9 oral cancer cells to gain insights about its functionality. The overexpression of PTCSC3 was authenticated by GRT-PCR analysis; it should about a 6.7- and 6.3-fold increase in PTCSC3 expression in SCC-1 and SCC-9 cells respectively (Figure $2 \mathrm{~A}$ ). The results of the cell viability assay showed that overexpression of PTCSC3 resulted in a significant decline in the proliferation of the SCC -1 and SCC -9 cells (Figure 2 B). The DAPI staining analysis showed that PTCSC3 overexpression triggers changes in the nuclear morphology of the SCC- 1 and SCC-9 cells such as nuclear blebbing and fragmentation indicative of apoptosis (Fig ure $3 \mathrm{~A}$ ). The annexin V/PI staining assays showed that the early and late apoptosis increased from $3.8 \%$ and $4.0 \%$ to $44.9 \%$ and $41.2 \%$ respectively, upon overexpression of PTCSC3 in SCC-1 cells. In case of SCC-9 cells, early and late apoptosis increased from $4.5 \%$ and $9.6 \%$ to $12.9 \%$ and $41.4 \%$ respectively upon PTCSC3 overexpression (Figure $3 \mathrm{~B}$ ). The expression of $\mathrm{Bax}$ and $\mathrm{BCl}-2$ was evaluated in the NC and pcDNA-PTCSC3 transfected SCC- 1 and SCC-9 cells. The results revealed remarkable enhancement of Bax and suppression of $\mathrm{BCl}-2$ in both the cell lines, further confirming the induction of apoptotic cell death (Figure 4).

\section{LncRNA PTCSC3 induces apoptosis in oral cancer cells}

After overexpression of PTCSC3 in SCC -1 and SCC-9 cells, ultrastructural analysis was performed

A

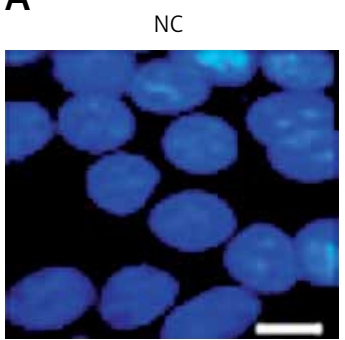

SCC-1

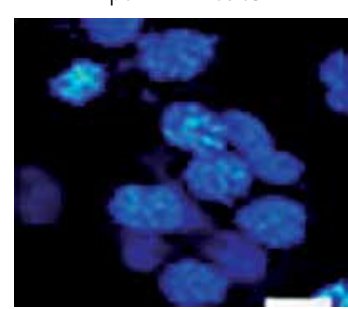

SCC-1

pCDNA-PTCSC3 by transmission electron microscopy (TEM). The TEM analysis showed the development of autophagic vesicles in both the SCC- 1 and SCC-9 cells indicative of autophagy (Figure $5 \mathrm{~A}$ ). The western blotting analysis showed that PTCSC3 overexpression caused a remarkable increase in LC3B-I and Beclin 1 expression, further confirming the induction of autophagy in human oral cancer cells (Figure $5 \mathrm{~B}$ ).

\section{LncRNA PTCSC3 inhibits invasion of oral cancer cells}

Transwell assays were performed to assess the effects of PTCSC 3 on the invasion of human SCC-1 and SCC-9 oral cancer cells. The results showed that PTCSC3 overexpression caused a significant decrease in invasion of the human SCC-1 and SCC -9 oral cancer cells. Invasion of the SCC- 1 and SCC -9 cells was inhibited by $62 \%$ and $69 \%$ respectively (Figure 6).

\section{Discussion}

It is well established that IncRNAs are aberrantly expressed in different cancer types. They play crucial roles in the development and progression of cancers. Studies have shown that IncRNAs may prove essential biomarkers to enable early detection of cancers $[17,18]$. Additionally, IncRNAs regulate cancer-related events such as apoptosis, autophagy and metastasis to name a few $[19,20]$. As such they may also exhibit therapeutic implications in the management of deadly cancers. This study for the first time examined the therapeutic implications of IncRNA PTCSC3 in human oral can-

SCC-9

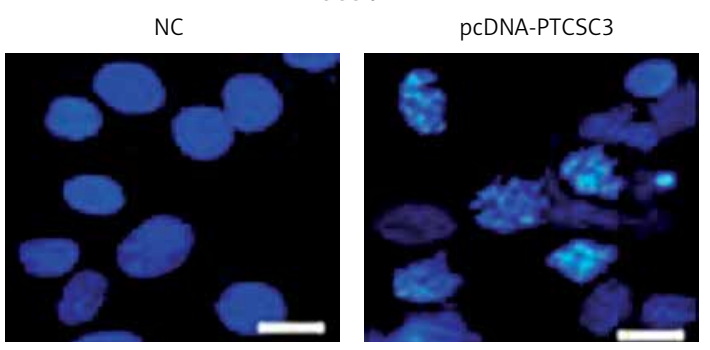

SCC-9

NC

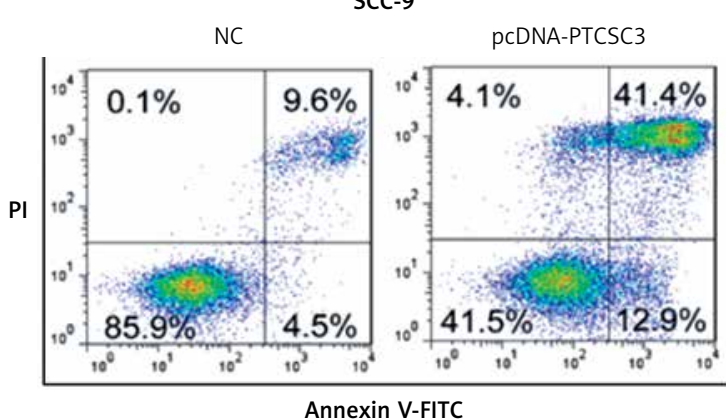

Annexin V-FITC

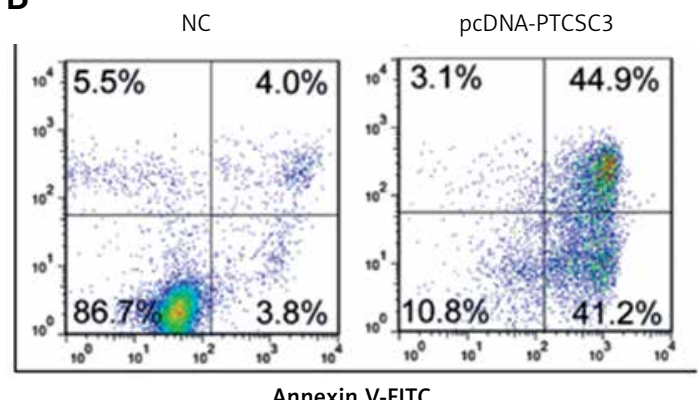

Figure 3. PTCSC3 induces apoptosis in oral cancer cells. DAPI staining (A) and Annexin V/PI staining (B) of NC and pcDNA-PTCSC3 transfected SCC-1 and SCC-9 oral cancer cells. The experiments were performed in triplicate 
SCC-1

NC

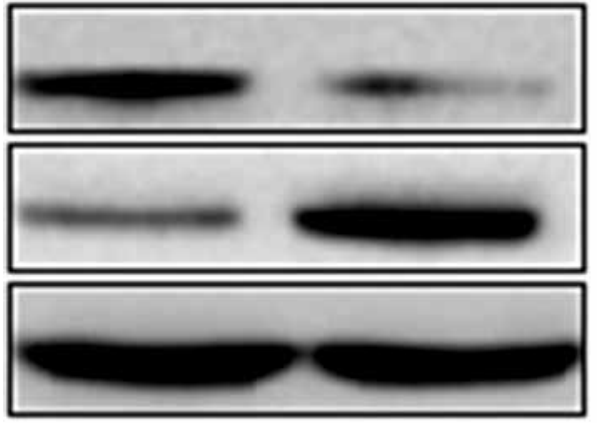

SCC-9 Bcl-2

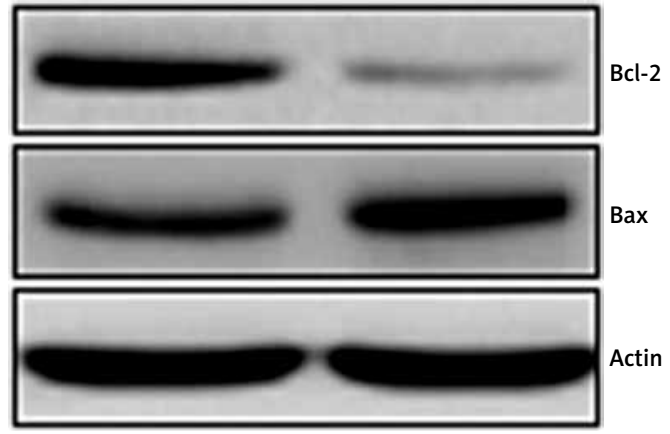

Figure 4. Expression of Bax and Bcl-2 in NC and pCDNA-PTCSC3 transfected SCC-1 and SCC-9 oral cancer cells. The experiments were performed in triplicate

A

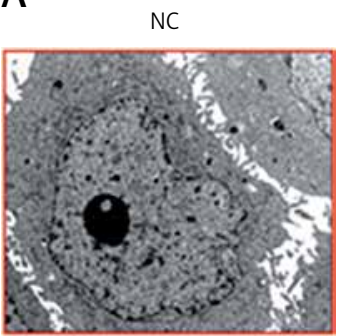

B

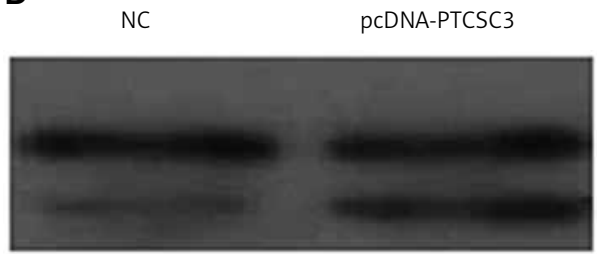

SCC-1

pCDNA-PTCSC3

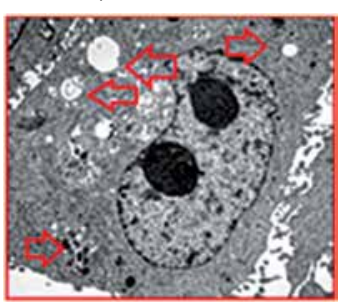

LC3B-I

LC3B-II

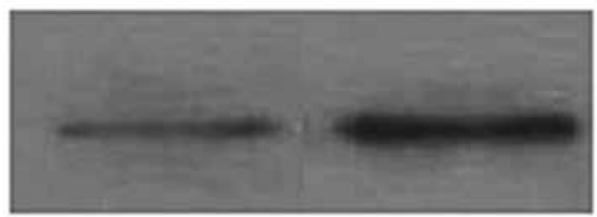

Beclin 1

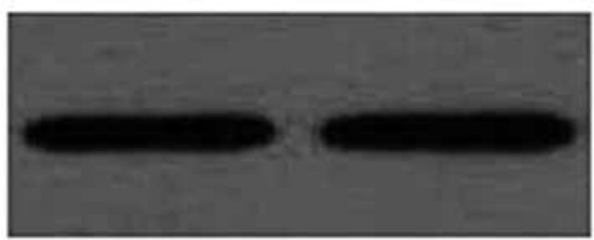

Actin
NC

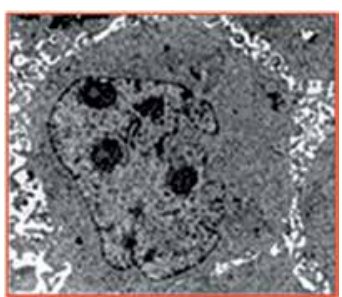

SCC-9

NC

pcDNA-PTCSC3
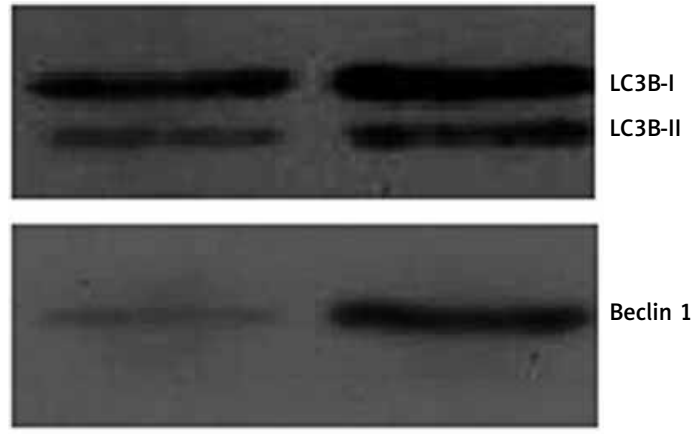

pCDNA-PTCSC3

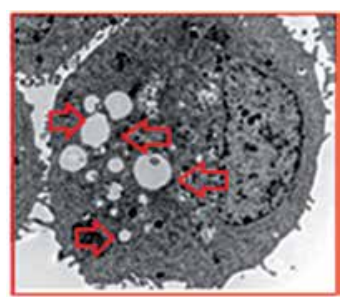

SCC-9 . 
SCC-1
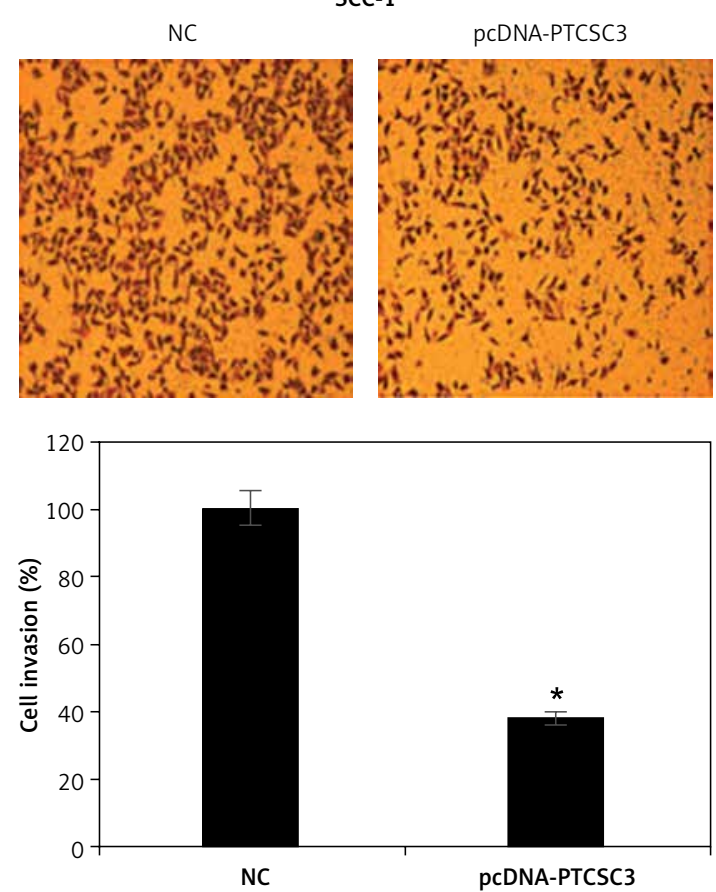

PCDNA-PTCSC3

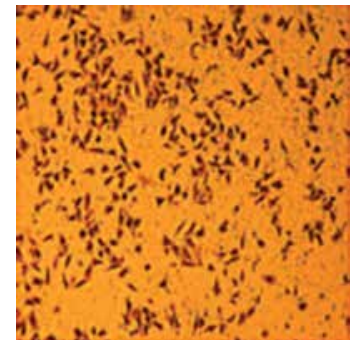

NC
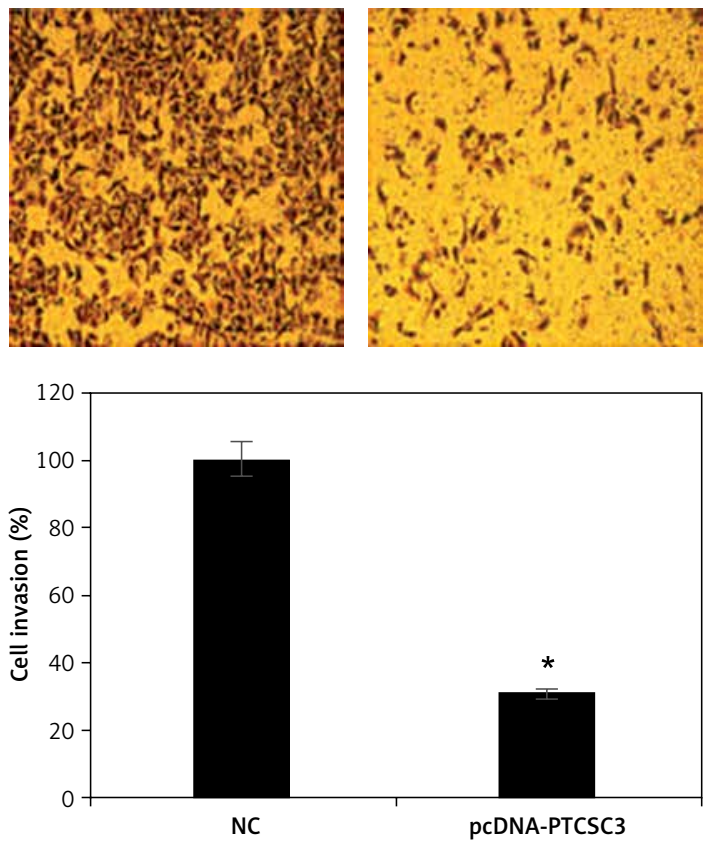

Figure 6. Transwell assays showing cell invasion in NC and pCDNA-PTCSC3 transfected SCC-1 and SCC-9 oral cancer cells. Individual experiments were performed in triplicate

PTCSC3 overexpression triggered both apoptosis and autophagy in human oral cancer cells $[21,22]$. This was accompanied by alteration of both apoptosis- and autophagy-related proteins. The apoptosis-related proteins such as Bax were increased while Bcl-2 was decreased. Similarly, autophagy proteins LC3B-II and Beclin 1 also exhibited a remarkable increase upon PTCSC3 overexpression. These findings are in agreement with several other studies wherein IncRNAs have been shown to trigger apoptosis or autophagy in human cancer cells. For example, IncRNA CASC2 overexpression suppressed the proliferation of human hepatocellular cancer and lung cancer cells by triggering apoptotic cell death $[23,24]$. In yet another study, IncRNA CCAT2 induced autophagy in human gastric cancer cells [25]. The invasion of the cancer cells from the site of origin to the adjacent tissues is critical for cancer metastasis [26]. In the present study we found that PTCSC3 suppresses the metastasis of human oral cancer. All considered, PTCSC3 may prove an essential therapeutic target for oral cancer treatment.

In conclusion, the findings of the present study show that IncRNA PTCSC3 was significantly suppressed in human oral cancer cells. Overexpression of PTCSC3 resulted in inhibition of the proliferation of human oral cancer cells via induction of apoptosis and autophagy. Additionally, PTCSC3 suppressed the metastasis of human oral cancer cells, indicative of the therapeutic implications of PTCSC3 in oral cancer.

\section{Conflict of interest}

The authors declare no conflict of interest.

\section{References}

1. Hangauer MJ, Vaughn IW, McManus MT. Pervasive transcription of the human genome produces thousands of previously unidentified long intergenic noncoding RNAs. PLoS Genet 2013; 9: e1003569.

2. Gutschner T, Diederichs S. The hallmarks of cancer: a long noncoding RNA point of view. RNA Biol 2012; 9: 703-19.

3. Liu X, Wang M, Cui Y. LncRNA TP73-AS1 interacted with miR-141-3p to promote the proliferation of non-small cell lung cancer. Arch Med Sci 2019; 15: 1547-54.

4. XU R, Han Y. Long non-coding RNA FOXF1 adjacent non-coding developmental regulatory RNA inhibits growth and chemotherapy resistance in non-small cell lung cancer. Arch Med Sci 2019; 15: 1539-46.

5. Kogo R, Shimamura T, Mimori K, et al. Long noncoding RNA HOTAIR regulates polycomb-dependent chromatin modification and is associated with poor prognosis in colorectal cancers. Cancer Res 2011; 71: 6320-6.

6. Yang Z, Zhou L, Wu LM, et al. Overexpression of long non-coding RNA HOTAIR predicts tumor recurrence in hepatocellular carcinoma patients following liver transplantation. Ann Surg Oncol 2011; 18: 1243-50.

7. Sirchia SM, Tabano S, Monti L, et al. Misbehaviour of XIST RNA in breast cancer cells. PLoS One 2009; 4: e5559.

8. Yao Y, Ma J, Xue Y, et al. Knockdown of long non-coding RNA XIST exerts tumor-suppressive functions in human glioblastoma stem cells by up-regulating miR-152. Cancer Lett 2015; 359: 75-86.

9. Li XL, Wu ZQ, Fu XB, Han WD. LncRNAs: insights into their function and mechanics in underlying disorders. Mutat Res Rev Mut Res 2014; 762: 1-21. 
10. Fan $M$, Li X, Jiang $W$, et al. A long non-coding RNA, PTCSC3, as a tumor suppressor and a target of miRNAs in thyroid cancer cells. Exp Ther Med 2013; 5: 1143-6.

11. Xia S, Ji R, Zhan W. Long noncoding RNA papillary thyroid carcinoma susceptibility candidate 3 (PTCSC3) inhibits proliferation and invasion of glioma cells by suppressing the Wnt/beta-catenin signaling pathway. BMC Neurology 2017; 17: 30.

12. Manikandan M, Rao AK, Arunkumar G, et al. Oral squamous cell carcinoma: microRNA expression profiling and integrative analyses for elucidation of tumourigenesis mechanism. Mol Cancer 2016; 15: 28-32.

13. Siegel RL, Miller KD, Jemal A. Cancer statistics, 2018. CA Cancer J Clin 2018; 68: 7-30.

14. Cancer Research Campaign. CancerStats. Oral cancer UK. UK: CRC; April 2005.

15. Conway D, Macpherson LMD, Warnakulasuriya KAAS, Ogden G, Stockton DL. Incidence of oral and oropharyngeal cancer in the United Kingdom (1990-1999) - current status, recent trends and intercountry comparisons. Oral Oncol 2006; 42: 586-92.

16. Banoczy J, Squier C. Smoking and disease. Eur J Dent Educ 2004; 8: 7-10.

17. Moran VA, Perera RJ, Khalil AM. Emerging functional and mechanistic paradigms of mammalian long non-coding RNAs. Nucleic Acids Res 2012; 40: 6391-400.

18. Pandey RR, Mondal T, Mohammad F, et al. Kcnq1ot1 antisense noncoding RNA mediates lineage-specific transcriptional silencing through chromatin-level regulation. Mol Cell 2008; 32: 232-46.

19. Zhang J, Zhang B, Wang T, Wang H. LncRNA MALAT1 overexpression is an unfavorable prognostic factor in human cancer: evidence from a meta-analysis. Int J Clin Exp Med 2015; 8: 5499-505.

20. Geng YJ, Xie SL, Li Q, Ma J, Wang GY. Large intervening noncoding RNA HOTAIR is associated with hepatocellular carcinoma progression. J Int Med Res 2011; 39: 2119-28.

21. Sun SY, Hail Jr N, Lotan R. Apoptosis as a novel target for cancer chemoprevention. J Natl Can Inst 2004; 96: 662-72.

22. Youn CK, Kim J, Park JH, Do NY, Cho SI. Role of autophagy in cisplatin-induced ototoxicity. Int J PediatrOtorhinolaryngol 2015; 79: 1814-9.

23. Fan JC, Zeng F, Le YG, Xin L. LncRNA CASC2 inhibited the viability and induced the apoptosis of hepatocellular carcinoma cells through regulating miR-24-3p. J Cell Biochem 2018; 119: 6391-7.

24. Li Q, Chen K, Dong R, Lu H. LncRNA CASC2 inhibits autophagy and promotes apoptosis in non-small cell lung cancer cells via regulating the miR-214/TRIM16 axis. RSC Adv 2018; 8: 40846-55.

25. Yu ZY, Wang Z, Lee KY, Yuan P, Ding J. Effect of silencing colon cancer-associated transcript 2 on the proliferation, apoptosis and autophagy of gastric cancer BGC823 cells. Oncol Letters 2018; 15: 3127-32.

26. Matouk IJ, Abbasi I, Hochberg A, Galun E, Dweik H, Akkawi M. Highly upregulated in liver cancer noncoding RNA I overexpressed in hepatic colorectal metastasis. Eur J Gastroen Hepat 2009; 21: 688-92. 